\title{
PERTE DE COMPOSÉS VOLATILS LORS DE LA DÉTERMINATION A L'ÉTUVE DE LA TENEUR EN MATIÈRE SĖGHE DES ENSILAGES
}

\author{
J.-P. DULPHY, C. DEMARQUILLY et Martine HENRY \\ avec la collaboration technique de Marie JAILlER, Jacqueline Jamor et L. L'Hotelier \\ Laboratoire des Aliments, \\ Centre de Recherches de Clermont Ferrand, I. N. R. A., \\ Theix, Saint Gonès Champanelle, 63110 Beaumont
}

\section{RÉSUMÉ}

Nous avons cherché à mettre au point une méthode de correction de la teneur en matière sèche des ensilages déterminéc à l'étuve qui tienne compte des différents produits volatils contenus dans ces ensilages et de leur taux de perte lors de l'étuvage.

Tout d'abord nous avons comparé les teneurs cn matière sèche déterminées dans deux étuves de type différent, mais réglées à la même température $\left(80^{\circ} \mathrm{C}\right)$. La différence de teneur en matière sèche est très faible et négligcable par rapport à la précision des déterminations de la digestibilité et cle l'ingestibilité des ensilages par les moutons.

La quantité de produits volatils perdue a été très comparable d'une étuve à l'autre. Les taux de perte des différents produits volatils ont varié selon les espèces végétales ou groupes d'espèces (graminées, luzernes, maîs) et selon le $\mathrm{pH}$ ou la composition chimique des ensilages. De plus, nous avons chiffré les pertes en acide lactique et alcools à l'étuve. vantes :

Compte tenu de cela, la quantité perdue à l'étuve peut être estimée par les équations sui-

$$
\begin{array}{ll}
\text { pour les graminées } & \mathrm{Y}=0,616 x_{1}+0,142 x_{2}+0,830 x_{3}+\mathrm{I}, 000 x_{4} \\
\text { pour les luzernes } & \mathrm{Y}=0,534 x_{1}+0,732 x_{3}+\mathrm{I}, 000 x_{4} \\
\text { pour les maïs } & \mathrm{Y}=0,382 x_{1}+0,106 x_{2}+0,782 x_{3}+\mathrm{I}, 000 x_{4}
\end{array}
$$

dans lesquelles $\mathrm{Y}$ est la quantité de matière sèche volatile perdue lors du séchage en $\mathrm{g}$ par $\mathrm{kg}$ de matière sèche non corrigée et $x_{1}, x_{2}, x_{3}$ et $x_{4}$ respectivement les teneurs en ammoniac, acide lactique, acicles gras volatils et alcools exprimés en g par $\mathrm{kg}$ de matière sèche non corrigée.

Pour corriger la teneur en matière sèche, il suffit alors de rajouter à $I$ ooo $g$ de " matière sèche étuve "la quantité $Y$.

Cette méthode de correction permet une bonne concordance avec celle de DEwAR et McDonald (I96I) qui utilisent une distillation dans le toluène. Elle est plus précise que celle proposée par Fatiaxoff et Gouet (I969) car elle tient compte des espèces végétales et, si on le veut, des caractéristiques propres de l'ensilage. De plus, elle inclut l'acide lactique et les alcools. Cependant, pour obtenir une meilleure concordance avec la méthode de Dewar et McDonald (I96r), il aurait fallu vraisemblablement doser l'acide formique dans les ensilages ayant reçu cet acide comme conservateur. 


\section{IN'TRODUC'TION}

La teneur en matière sèche des ensilages est habituellement déterminée après séchage à l'étuve. Or, on sait depuis longtemps que ce séchage entraîne des pertes de produits volatils (ScHoch, I949; Colovos ot al., 1957). Il faut donc corriger la teneur déterminée à l'étuve (FAtianorF et Goust, I969) ou utiliser une autre méthode de détermination. La méthode la plus précise qui a été proposée est celle de McDonald et Dewar (I960) complétée par IEwar et McDonald (I96I). Flle consiste à déterminer l'eau contenue dans l'ensilage par une distillation dans le toluène. Elle a été reprise en particulier par FENNER et BARNEs (1965) et BRAHMAKSHATRIYA et DONKER (I97I). Cependant, elle présente toujours quelques inconvénients pour devenir une méthode de routine (AERTs et al., 1974) ; impossibilité de déterminer un grand nombre de teneurs en matière sèche chaque jour, nécessité d'utiliser de petits échantillons, danger pour la personne qui manipule. C'est pourquoi nous avons cherché à mettre au point une méthode de correction inspirée de celle de Schoch (I949) et de FatianofF et Gouet (I969) mais tenant compte plus précisément des différents produits volatils contenus dans les ensilages. Dans un premier temps, nous avons comparé les teneurs en matière sèche déterminées à même température dans 2 étuves différentes pour savoir si la teneur en matière sèche déterminée par séchage était liée au type d'étuve. Puis, nous avons mesuré l'importance des pertes des différents produits volatils des ensilages et leurs facteurs de variations. Enfin, nous avons examiné la possibilité de corriger les teneurs déterminées à l'étuve en y rajoutant les produits connus perdus à l'étuve et en comparant le résultat obtenu à la teneur en matière sèche déterminée après distillation dans le toluène.

\section{MATÉRIEL E'T MÉTHODES}

\section{Ensilages}

Nous avons, au cours de 2 années successives (1972 et I973), utilisé au total I 2 I ensilages de graminées, de luzerne et de maîs (plante entière) se répartissant comme il est indiqué dans le tableau 1 .

Les maïs avaient une teneur en matière sèche faible cn 1972 (environ 22 p. Ioo) et plus élevée en 1973 (environ 36 p. Ioo).

\section{Mesures effectuées}

a) Nous avons comparé les tencurs en matière sèche déterminées dans 2 étuves clifférentes :

- une étuve A de petite capacité, non ventilée ( $t=80^{\circ} \mathrm{C}, 48$ heures) et utilisée au laboratoire pour déterminer sur $200 \mathrm{~g}$ de matière fraîche la teneur en matière sèche de l'échantillon représentatif de l'ensilage distribué pendant la période de 6 jours ( 2 repas) de mesure de l'ingestibilité et de la digestibilité par des moutons. Sur cet échantillon ont été déterminées au laboratoire, la composition chimique et les caractéristiques fermentaires de l'ensilage frais ;

- une étuve $\mathrm{B}$ ventilée de grande capacité $\left(t=80^{\circ} \mathrm{C}, 24\right.$ heures) utilisée pour (léterminer sur I $\mathrm{kg}$ de matière frâ̂che la tencur en matière sèche des ensilaçes offerts à chaque repas aux moutons.

Pour cette comparaison les I 2 I ensilages ont été utilisés et tous ont été au préalable broyés pour éviter les difficultés d'échantillonnage. 
TABLEAU I

Nombre d'ensilages sur lesquels ont été effectuées les différentes mesures

\begin{tabular}{|c|c|c|c|c|c|}
\hline \multirow[b]{2}{*}{ Catégorie d'ensilages } & \multirow[b]{2}{*}{ Total } & \multicolumn{2}{|c|}{ Annce } & \multicolumn{2}{|c|}{$\begin{array}{l}\text { Ensilages dont la teneur en } \\
\text { matière sèche a été déterminée } \\
\text { dans le toluène }\end{array}$} \\
\hline & & 1972 & 1971 & $\begin{array}{l}\text { Ensilages préparés } \\
\text { avec addition } \\
\text { d'acide formique }\end{array}$ & $\begin{array}{c}\text { Ensilages préparés } \\
\text { sans conservateur } \\
\text { ou sans } \\
\text { acide formique }\end{array}$ \\
\hline $\begin{array}{l}\text { Graminces } \\
\text { Luzerne } \\
\text { Maïs }\end{array}$ & $\begin{array}{l}74 \\
21 \\
26\end{array}$ & $\begin{array}{r}39 \\
8 \\
16\end{array}$ & $\begin{array}{l}35 \\
13 \\
10\end{array}$ & $\begin{array}{r}22 \\
13 \\
0\end{array}$ & $\begin{array}{r}19 \\
0 \\
15\end{array}$ \\
\hline
\end{tabular}

b) Évaluation des pertes en ammoniaque, acides organiques (lactique, acétique, propionique, butyrique) et alcools (méthanol, éthanol, propanol, butanol) par comparaison de leurs teneurs avant et après séchage. Pour cela nous avons utilisé 24 ensilages de graminées, I 8 de luzerne et 23 de maïs, séchés dans l'étuve A. De plus, io des ensilages de chaque catégorie ont été étudiés après passage dans l'étuve B. Nous avons dosé l'acide lactique des ensilages avant et après séchage bien que FATIANOFF et GOUET (I969) l'aient considéré comme non volatil car McDonald et Dewar (1960) et Wilson et al. (1964) ont montré qu'il était partiellement volatil.

c) Comparaison des teneurs en matière sèche mesurées dans l'étuve $\mathrm{A}$ et après distillation dans le toluène. Elle a porté sur $4 \mathrm{I}$ ensilages de graminées, I 3 de luzerne et ${ }_{5}$ de maïs.

\section{Analyses}

Les analyses des échantillons frais ont été effectuées soit sur le jus extrait par pression (pH, teneur en ammoniaque, acides gras volatils et alcools) soit sur le jus provenant d'une macération de $50 \mathrm{~g}$ d'ensilage frais dans $45^{\circ} \mathrm{g}$ d'eau pendant $\mathrm{I} 6$ heures à $+4^{\circ} \mathrm{C}$ (acide lactique). Les analyses des échantillons séchés à l'étuve ont été effectuées sur le jus provenant d'une macération de $50 \mathrm{~g}$ d'ensilage sec dans $25^{\circ} \mathrm{g}$ d'cau pendant 16 heures à $+4^{\circ} \mathrm{C}$ (ammoniaque, acides organiques, alcools).

L'ammoniaque a été dosé par la méthode de Conway (I957) et l'acide lactique par la méthode de BARKER et Summerson (I94I).

Les acides gras volatils ont été dosés par chromatographie en phase gazeuse selon la méthode de Rigaud et Journet (I970) modifiée. En effet, nous n'utilisons plus qu'une seule colonne de I,50 m contenant un mélange de 80 p. Ioo de Carbowax $20-\mathrm{M}$ et 20 p. Ioo de chromo-

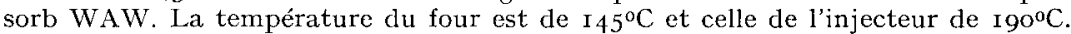

Les alcools ont été dosés également par chromatographie en phase gazeuse après distillation du jus d'ensilage saponifié (par la soude). Nous avons utilisé ensuite la même technique que pour le dosage des acides gras volatils mais la température du four était fixée à $80^{\circ} \mathrm{C}$ et celle de l'injecteur à $125^{\circ} \mathrm{C}$.

\section{Détermination de la teneur en matière sèche}

- A l'étuve : par peséc avant et après séchage.

- A l'étuve après correction : en rajoutant les produits volatils perdus dans l'étuve, soit réellement mesurés, soit estimés à partir des teneurs des ensilages en différents produits volatils et du pourcentage de perte à l'étuve de ces produits.

- Après distillation dans le toluène (DEwaR et McDonalo, I96i) et correction pour les produits volatils comme suit :

- acides après dosage à la soude et utilisation de la formule de Dewar et McDonald, (r96r) ;

- alcools en tenant compte du volume occupé par l'éthanol et de son coefficient de volati. lisation dans le toluène ( $86 \mathrm{p}$. Ioo pour les Io mesures effectuées dans notre laboratoire). 


\section{RÉSULTATS \\ Comparaison des teneurs en matière sèche mesurées dans 2 étuves différentes (tabl. 2)}

Pour les I2 I ensilages comparés, la teneur moyenne en matière sèche déterminée dans 1'étuve $\mathrm{A}$ a été de $2 \mathrm{I}, \mathrm{I} 6$ contre $2 \mathrm{I}, 27$ dans l'étuve $\mathrm{B}$. La différence est très faible (o,II point soit $0,52 \mathrm{p}$. IOO) bien qu'elle soit significative pour certaines catégories d'ensilage ; elle est négligeable par rapport à la précision des déterminations de la digestibilité $( \pm 2,5$ p. roo) et de l'ingestibilité ( \pm Io p. IOO) des ensilages par les moutons.

\section{TABLEAU 2}

Différence dans la détermination de la teneur en matière sèche des ensilages selon le type d'étuve utilisé

\begin{tabular}{|c|c|c|c|c|c|}
\hline & - & Nombre & Teneur en & ière Sèche & Seuil \\
\hline & & d'échantillons & Etuve A & Etuve $B$ & de la différence \\
\hline \multirow{2}{*}{ Graminées } & 1972 & 39 & $\simeq 0,18$ & $20,4^{\prime}+$ & $\mathrm{P}<0,05$ \\
\hline & 1973 & 35 & 17,13 & 17,05 & NS \\
\hline \multirow{2}{*}{ Luzerne } & 1972 & 8 & $21,6: 3$ & 21,86 & NS \\
\hline & 1973 & 13 & 21,67 & 21,85 & $P<0,05$ \\
\hline \multirow{2}{*}{ Maïs } & 1972 & 16 & 22,21 & 22,29 & NS \\
\hline & 1973 & 10 & 36,10 & 36,38 & NS \\
\hline
\end{tabular}

Étude du taux de pertes des différents composés volatils des ensilages

En comparant les teneurs en produits volatils déterminées sur les ensilages frais et après séchage à l'étuve, nous avons calculé les quantités de produits volatils perdues lors du séchage et les taux de pertes de ces différents produits.

La quantité de produits volatils perdue suivant le type d'étuve utilisée n'a pas été significativement différente pour les graminées (tabl. 3). Pour les luzernes et les maïs, la différence a été très faible : $2 \mathrm{~g}$, en accord avec le fait que les teneurs en matière sèche déterminées dans ces deux types d'étuve ont été elles-mêmes très voisines.

Le taux de pertes de l'ammoniac a varié beaucoup. Il a été en moyenne de 50,8 p. roo pour les 65 ensilages étudiés (tabl. 4). Sauf pour les ensilages de maìs, le taux de pertes de ce composé (tabl. 5) a été d'autant plus important que sa teneur était élevée (ensilages de luzerne), le pH élevé et la teneur en acide lactique faible 
(ensilages de graminées et de luzerne) ou que les teneurs en acides gras volatils totaux étaient faibles (ensilages de graminées). On peut noter que pour un $\mathrm{pH}$ ou une teneur en acide lactique donné, le taux de pertes de l'ammoniac est plus important pour les ensilages de graminées que pour ceux de luzerne et de maïs.

\section{TABI,EAU 3}

Différence cntre les pertes (en $\mathrm{g}$ par $\mathrm{kg}$ de matière sèche) de composés volatils (sauf alcools et acides propionique et butyrique) selon le type d'étuve utilisé pour la détermination de la teneur en matière sèche

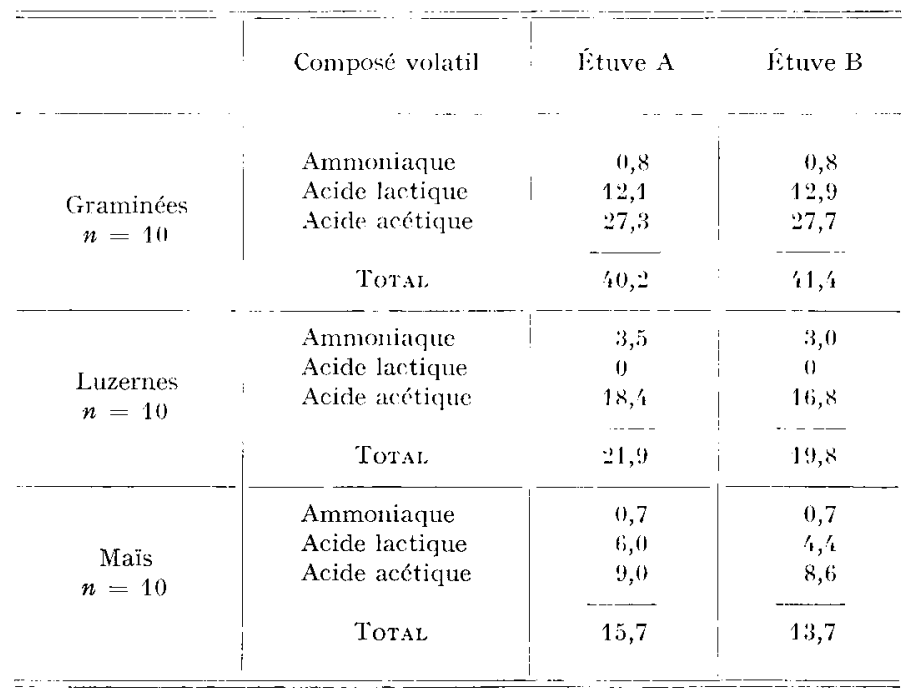

Le séchage n'a entraîné des pertes d'acide lactique que dans les ensilages de graminées et de maïs. Dans ces derniers, le taux de pertes de cet acide a été en moyenne de I2, 4 p. Ioo, mais a varié beaucoup (tabl. 4). Quoique très variable, ce taux de pertes n'a été lié ni au $\mathrm{pH}$, ni aux teneurs en ammoniaque ou en acides organiques de l'ensilage.

Le taux de pertes de l'acide acétique a été en moyenne de $78,7 \mathrm{p}$. Ioo pour les 65 ensilages, avec de faibles variations. Le taux de pertes de l'acide butyrique n'a pu être mesuré que sur 7 ensilages; il a été proche $(83,2$ p. Ioo en moyenne) de celui de l'acide acétique. Les autres ensilages contenaient trop peu d'acide butyrique, de même que tous les ensilages contenaient trop peu d'acide propionique pour qu'il soit possible de doser correctement les traces de ces acides restant après séchage.

Pour les diverses catégories d'ensilage, le taux de pertes de l'acide acétique a été d'autant plus important que le $\mathrm{pH}$ et la teneur en ammoniaque étaient faibles (bien que les liaisons ne soient pas significatives pour les ensilages de mais), et que les teneurs en acides lactique et acétique étaient élevées. Il est intéressant de constater que pour un même $\mathrm{pH}$ les taux de pertes de l'acide acétique dans les ensilages de graminées et de luzernes ne sont pas significativement différents et sont en moyenne 


\section{TABLEAU 4}

Pertes des produits volatils à l'étuve (Étuve A)

\begin{tabular}{|c|c|c|c|c|}
\hline & Composés volatils & $\begin{array}{c}\text { Teneur en } g / \mathrm{kg} \\
\text { de matière sèche } \\
\text { non corrigée }\end{array}$ & $\begin{array}{c}\text { Quantité perdue } \\
\text { dans l'étuve en g/kg } \\
\text { de matière sèche } \\
\text { non corrigée }\end{array}$ & $\begin{array}{c}\text { Taux de pertes } \\
(\%)\end{array}$ \\
\hline \multirow{7}{*}{$\begin{array}{c}\text { Graminées } \\
n=24 \\
\mathrm{pH}=4,19 \pm 0,22\end{array}$} & Ammoniaque & $\begin{array}{c}2,26 \\
(1,15 \stackrel{\text { à }}{3}, 65)\end{array}$ & $\begin{array}{c}1,4 \\
(0,27 \stackrel{\text { à }}{3,01)}\end{array}$ & $61,6 \pm 22,4$ \\
\hline & Acide lactique & $\begin{array}{c}77,3 \\
(46,5 \text { à } 126,2)\end{array}$ & $\begin{array}{c}10,9 \\
(0,1 \text { à } 36,4)\end{array}$ & $14,2 \pm 8,5$ \\
\hline & Acide acétique & $\begin{array}{c}23,2 \\
(11,0 \text { à } 44,1)\end{array}$ & $\begin{array}{c}19,7 \\
(8,4 \text { à } 39,5)\end{array}$ & $83,0 \pm 7,5$ \\
\hline & Acide propionique & $\begin{array}{c}0,5 \\
(0,0 \text { à } 2,0)\end{array}$ & non déterminé & non déterminé \\
\hline & Acide butyrique & $\begin{array}{c}\text { 4,7 } \\
(0,0 \stackrel{\text { à } 30,9)}{ }\end{array}$ & $\begin{array}{c}\text { déterminé } \\
\text { sur } 2 \text { ensilages }\end{array}$ & $87,2(n=2)$ \\
\hline & Alcools & $\begin{array}{c}14,5 \\
(3,2 \text { à } 62,3)\end{array}$ & 14,5 & 100,0 \\
\hline & Total & & 50,4 & \\
\hline \multirow{7}{*}{$\begin{array}{c}\text { Luzerne } \\
n=17 \\
\mathrm{pH}=4,60 \pm 0,28\end{array}$} & Ammoniaque & $\begin{array}{c}5,10 \\
(2,42 \text { à } 11,16)\end{array}$ & $\begin{array}{c}3,1 \\
(0,73 \text { à } 10,56)\end{array}$ & $53,4 \pm 19,5$ \\
\hline & Acide lactique & $\begin{array}{c}53,4 \\
(11,4 \text { à } 87,4)\end{array}$ & $(-9,6)$ & $-4,3 \pm 11,2$ \\
\hline & Acide acétique & $\begin{array}{c}24,9 \\
(18,4 \text { à } 36,4)\end{array}$ & $\begin{array}{c}18,3 \\
(11,5, \mathbf{a} 29,9)\end{array}$ & $73,2 \pm 9,5$ \\
\hline & Acide propionique & $\begin{array}{c}1,4 \\
(0,0 \text { à } 3,7)\end{array}$ & non déterminé & non déterminé \\
\hline & Acide butyrique & $\begin{array}{c}15,3 \\
(0,0 \text { à } 78,9)\end{array}$ & $\begin{array}{c}\text { déterminé } \\
\text { sur } 5 \text { ensilages }\end{array}$ & $81,6(n=5)$ \\
\hline & Alcools & $\begin{array}{c}12,0 \\
(4,3 \text { à } 20,9)\end{array}$ & 12,0 & 100,0 \\
\hline & Total & & 44,4 & \\
\hline \multirow{7}{*}{$\begin{array}{c}\text { Maïs } \\
n=24 \\
\mathrm{pH}=4,13 \pm 0,19\end{array}$} & Ammoniaque & $\begin{array}{c}2,42 \\
(1,70 \text { à } 3,27)\end{array}$ & $\begin{array}{c}0,9 \\
(0,24 \text { à } 1,80)\end{array}$ & $38,2 \pm 11,7$ \\
\hline & Acide lactique & $\begin{array}{c}60,4 \\
(32,3 \text { à } 95,2)\end{array}$ & $\begin{array}{c}6,6 \\
(-3,6 \text { à } 23,9)\end{array}$ & $10,6 \pm 8,4$ \\
\hline & Acide acétique & $\begin{array}{c}17,9 \\
(8,6 \text { à } 30,1)\end{array}$ & $\begin{array}{c}14,6 \\
(5,1 \text { à } 30,8)\end{array}$ & $78,2 \pm 11,2$ \\
\hline & Acide propionique & 0,0 & 0,0 & - \\
\hline & Acide butyrique & 0,0 & 0,0 & 一 \\
\hline & Alcools & $\begin{array}{c}32,5 \\
(2,5 \text { à } 86,1)\end{array}$ & 32,5 & 100,0 \\
\hline & TOTAL & & 54,6 & \\
\hline
\end{tabular}




\section{TABLEAU 5}

Infuence du $p H$, des teneurs en ammoniaque, acide lactique et acides organiques

sur le taux de pertes ( $Y$ en p. Ioo) des différents composés volatils

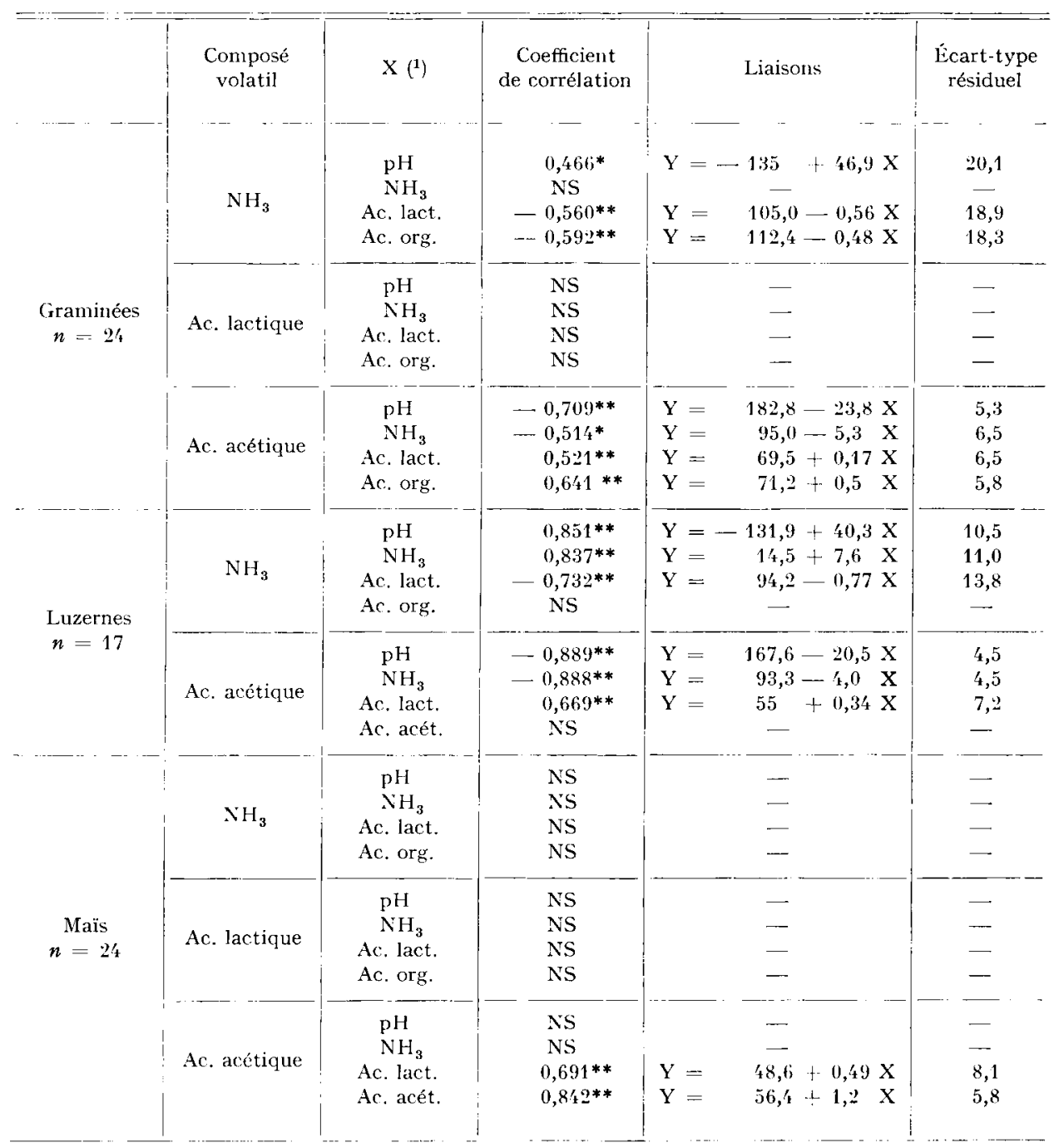

(1) pH exprimé en unité pH; Ammoniaque et acides en g/kg de matière sèche non corrigée. 
inférieurs de 5 à 6 points pour les ensilages de maïs, ce qui d'ailleurs ne correspond pas non plus à une différence significative.

Le taux de pertes de l'alcool a été de Ioo p. roo; nous n'avons en effet jamais retrouvé d'alcool dans les ensilages séchés à l'étuve.

Finalement pour les 65 ensilages étudiés les quantités perdues de produits volatils connus ont représenté en moyenne $50,8 \mathrm{~g}$ par $\mathrm{kg}$ de matière sèche non corrigée.

\section{Proposition d'une méthode de correction de la teneur en matière sèche déterminée par séchage}

Nous avons donc une idée relativement précise des taux de pertes à l'étuve des différents composés volatils des ensilages et de leurs facteurs de variation. Nous avons reporté les valeurs de ces taux dans le tableau 6 . Pour les pertes en acide propionique et en acide butyrique nous avons pris les valeurs concernant l'acide acétique.

Connaissant la teneur d'un ensilage en produits volatils et les taux de pertes de ces derniers à l'étuve, il est facile de calculer les pertes de matière sèche volatile lors du séchage. Pour corriger la teneur en matière sèche déterminée à l'étuve, il suffit alors de rajouter ces pertes. Par exemple, si la perte de matière sèche volatile lors du séchage est de $5^{\circ} \mathrm{g}$ par $\mathrm{kg}$ de matière sèche non corrigée, on sait que I ooo $\mathrm{g}$ de matière sèche non corrigée correspondent à I $05^{\circ} \mathrm{g}$ de matière sèche corrigée. La teneur en matière sèche déterminée à l'étuve est donc sous-estimée de $\frac{\text { I } 050}{\text { I } 000}=\mathrm{I}, 050$ d'où :

teneur en matière sèche réelle $=$

I,050 $\times$ teneur en matière sèche déterminée à l'étuve.

Pour le calcul des pertes à l'étuve dans les conditions de séchage voisines des nôtres (étuve réglée à $80^{\circ} \mathrm{C}$, séchage durant 24 ou 48 heures suivant que l'étuve est ventilée ou non), on pourra :

- soit utiliser les taux de pertes donnés dans le tableau 6 en fonction du $\mathrm{pH}$ qui est le principal facteur de variation du coefficient de volatilité de l'ammoniac et de l'acide acétique dans les ensilages de graminées et de luzerne, et en fonction de la teneur en acide acétique pour le taux de pertes de ce dernier dans les ensilages de maïs. On supposera que le taux de pertes des acides propionique et butyrique est identique à celui de l'acide acétique ;

- soit plus simplement en utilisant les taux de pertes moyens, ce qui donne les formules suivantes:

$$
\begin{array}{ll}
\text { Graminées } & \mathrm{Y}=0,6 \mathrm{I} 6 x_{1}+0,142 x_{2}+0,830 x_{3}+\mathrm{I}, 000 x_{4} \\
\text { Luzernes } & \mathrm{Y}=0,534 x_{1}+0,732 x_{3}+\mathrm{I}, 000 x_{4} \\
\text { Maïs } & \mathrm{Y}=0,382 x_{1}+0,106 x_{2}+0,782 x_{3}+\mathrm{I}, 000 x_{4}
\end{array}
$$

dans lesquels $\mathrm{Y}$ est la quantité de matière sèche volatile perdue lors du séchage en $\mathrm{g}$ par $\mathrm{kg}$ de matière sèche non corrigée et $x_{1}, x_{2}, x_{3}, x_{4}$ respectivement les teneurs en ammoniaque, acide lactique, acides gras volatils et alcools exprimés en $\mathrm{g}$ par $\mathrm{kg}$ de matière sèche non corrigée.

Ce sont ces formules que nous avons appliquées dans la suite de cette étude car 
pour nos ensilages, la prise en compte des variations des coefficients de volatilité n'apporte que de faibles différences dans l'estimation des pertes à l'étuve.

Dans ces pertes nous n'avons pas inclus les pertes d'acide formique parce que nous n'avons pas dosé cet acide. Il aurait fallu en tenir compte car il est classiquement trouvé dans les ensilages, notamment s'ils ont été préparés avec addition d'acide formique comme conservateur (WAL,Do et al., I97I).

\section{TABI,EAU 6}

Influence du $p H$ ou de la teneur en acide acétique sur les taux de pertes ( $\mathrm{p}$. Ioo) de différents composés

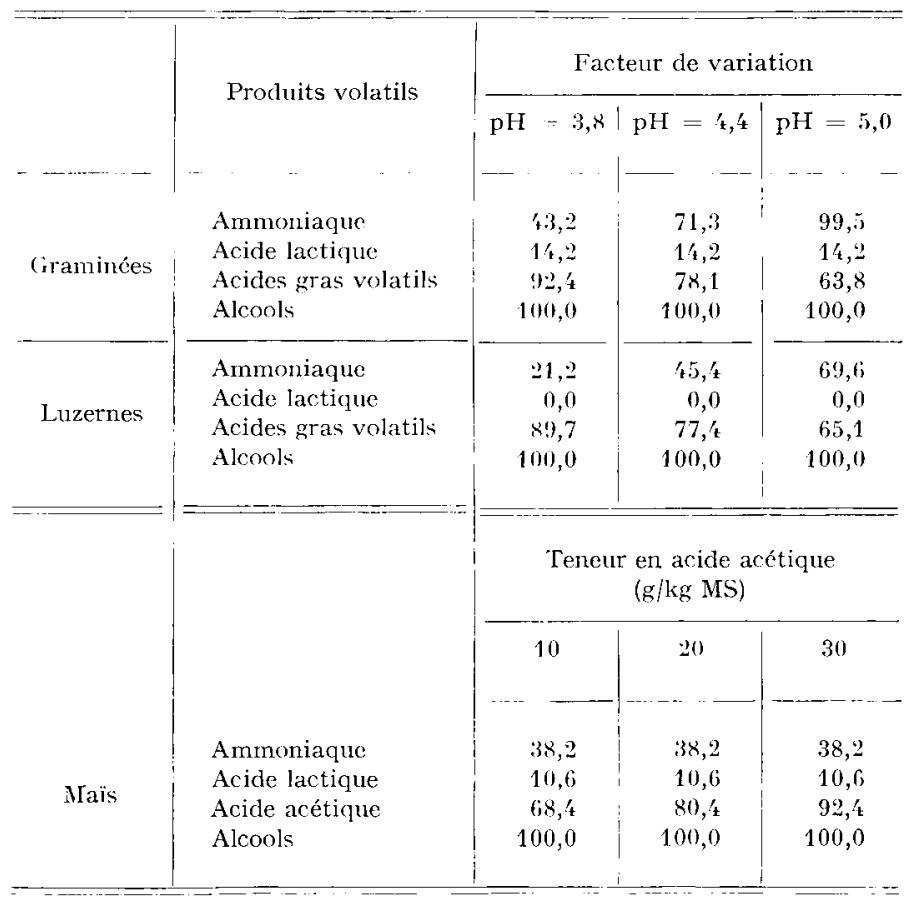

\section{Étude de la validité de la méthode proposée}

Nous avons mesuré par la méthode au toluène la teneur en matière sèche de $3^{8}$ (I4 graminées, II luzernes et I3 mais) des 65 échantillons d'ensilage sur lesquels l'ammoniaque, l'acide lactique, les acides gras volatils et les alcools ont été dosés avant et après séchage. Pour ces 38 ensilages, il existe une très bonne concordance entre la teneur en "matière sèche toluène " et la teneur en " matière sèche étuve corrigée " pour les pertes réellement observées (différences de teneurs avant et après étuvage) lors du séchage à l'étuve (fig. I). En moyenne, la teneur en " matière sèche étuve corrigée " est cependant légèrement plus faible que celle déterminée au toluène : 23,73 contre 23,83 pour les 38 échantillons, la différence n'étant cependant significative $(\mathrm{P}<0,05)$ que pour les I4 graminées : 23,82 contre $24, \mathrm{I} 2)$. 
De même, il existe, pour l'ensemble des 69 échantillons dont nous avons mesuré la teneur en matière sèche par la méthode au toluène, une bonne concordance entre la teneur en "matière sèche toluène " et la teneur en "matière sèche étuve corrigée " pour les quantités estimées (selon nos formules) de produits volatils perdus

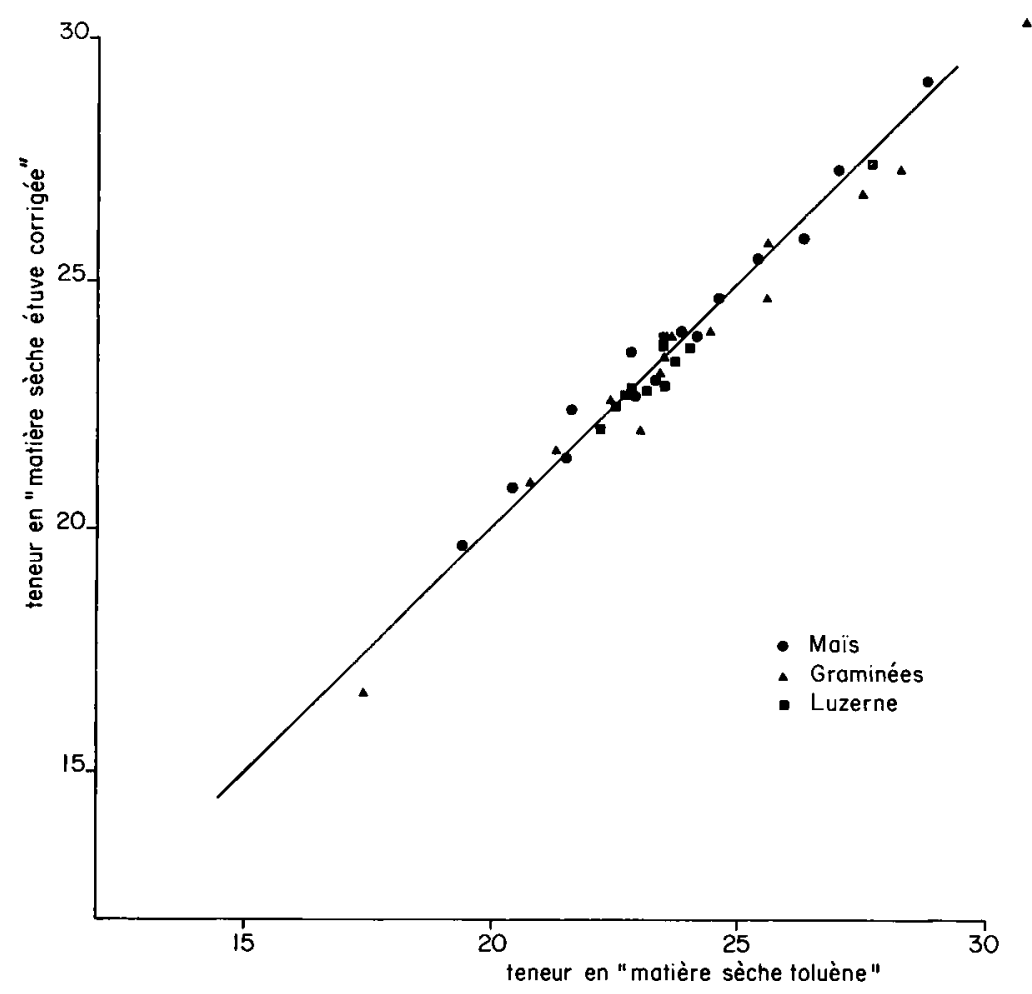

$\mathrm{FiG}_{\mathrm{I}}$ I. - Liaison entre teneurs en "matiere sìche toluine" et "matière séche étuve corrigée "selon nos lormules

lors du séchage, respectivement en moyenne pour les 69 échantillons 22,73 contre 22,64, la différence (o,09 point, soit 0,4 p. I00) n'étant pas significative (tab1. 7). On peut cependant observer que l'accord est meilleur pour les échantillons d'ensilages préparés sans addition d'acide formique, respectivement pour la " matière sèche toluène " et la "matière sèche étuve corrigée " (selon nos formules) 23,33 contre 23,37 p. Ioo pour $n=34$, soit une différence de 0,04 point (o,I 7 . IOo) et pour ceux préparés avec addition d'acide formique $22, I_{5}$ contre $2 \mathrm{I}, 93$, soit une différence de 0,22 point ( $\mathrm{I}$ p. IOO), ce qui correspond à une sous-estimation d'environ Io $\mathrm{g}$ par $\mathrm{kg}$ de matière sèche des pertes de produits volatils lors du séchage à l'étuve.

Nous avons également recalculé la teneur en "matière sèche étuve corrigée " des 56 ensilages étudiés par C. Demarquiliy (1973). Les alcools, non dosés, n'ont pas été pris en compte dans la méthode "toluène " ou "étuve corrigée ". Dans ce cas, encore il y a une bonne concordance dans les résultats obtenus, respectivement en moyenne $2 \mathrm{I}, 77$ contre $2 \mathrm{I}, 6 \mathrm{I}$. 


\section{TABLEAU 7}

Comparaison entre les teneurs

en matière sèche déterminée soit à partir de la distillation dans le toluène (1), soit après avoir rajouté à la matiève sèche déterminée à l'étuve les quantités estimées de produits volatils perdus $\left(^{2}\right)$

\begin{tabular}{|c|c|c|c|}
\hline & $\begin{array}{l}\text { Teneur en } \\
\text { matière sèche } \\
\text { déterminée } \\
\text { a l'étuve }\end{array}$ & $\begin{array}{l}\text { Teneur en } \\
\text { matière sèche } \\
\text { déterminée } \\
\text { selon (l) }\end{array}$ & $\begin{array}{l}\text { Teneur en } \\
\text { matière sèche } \\
\text { corrigée } \\
\text { selon }\left({ }^{2}\right)\end{array}$ \\
\hline 41 graminées & 21,05 & 22,14 & 21,97 \\
\hline 13 luzernes & 22,28 & 23,33 & 23,17 \\
\hline 15 Mais & 22,29 & 23,83 & 23,99 \\
\hline ГOTAL $(n=-69)$ & 21,55 & 22,73 & $22,6 / 4$ \\
\hline $\begin{array}{l}\text { Hnsilages sans } \\
\text { acide formique } \\
\qquad(n=34)\end{array}$ & 21,98 & 23,32 & 23,37 \\
\hline $\begin{array}{l}\text { linsilages avec } \\
\text { acide formique } \\
\qquad(n=35)\end{array}$ & 21,13 & 22,15 & 21,93 \\
\hline $\begin{array}{l}\text { Ensilages étudiés } \\
\text { par DeMarovilly } \\
(1973) \\
(n=56 \text {; alcools } \\
\text { non dosés })\end{array}$ & 20,66 & 21,77 & 21,61 \\
\hline
\end{tabular}

\section{DISCUSSION}

La méthode au toluène de détermination de la teneur en matière sèche des ensilages proposée par DEWAR et McDonALD (I96I) n'étant pas adaptée au dosage en série d'un grand nombre d'échantillons chaque jour, nous avons cherché à mettre au point une méthode d'estimation des produits volatils perdus lors du séchage à $80^{\circ} \mathrm{C}$ afin de pouvoir corriger la teneur en matière sèche déterminée à l'étuve. Les teneurs en matière sèche déterminées dans les deux types d'étuves utilisées étant comparables, on peut supposer que nos résultats sont extrapolables à d'autres types d'étuve dans la mesure où leur température de séchage est de $80^{\circ} \mathrm{C}$.

Prenant comme référence la méthode de DEwar et McDonald, la teneur en matière sèche déterminée à l'étuve réglée à $80^{\circ} \mathrm{C}$ de 69 échantillons de différents ensilages a été en moyenne sous-estimée de 5,47 p. Ioo (de 3,3 à I 4,4 p. Ioo selon les échantillons si on excepte un échantillon dont la teneur en matière sèche "étuve " a été supérieure de $3.9 \mathrm{p}$. Ioo à celle déterminée au toluène). Cette valeur moyenne est tout à fait comparable à celles trouvées par BRAHMAKSHATRIYA (I97I), IAARSEN 
et JoNEs (I973), AERTs et al. (I974) soit en moyenne respectivement 3,4 à 8,5, 6, I à 8, I et 7,0 à 9,5 p. Ioo selon la température de séchage employée.

Comme l'ont fait Schoch (I949) et FA'TIANOFF et GoUET (I969), nous proposons de corriger la teneur en matière sèche déterminée à l'étuve en estimant les pertes de matière sèche volatile lors du séchage. Cette estimation suppose la connaissance de la teneur de l'ensilage en composés volatils et du taux de pertes de chacun de ces composés. Le dosage de ces derniers à l'exception de l'acide formique et parfois des alcools, est déjà classiquement effectué pour connaître la qualité de conservation de l'ensilage. Il suffit donc de connaître le taux de pertes des différents composés volatils.

Les taux de perte de l'ammoniaque et des acides gras volatils que nous avons trouvés sont en moyenne tout à fait comparables à ceux trouvés par d'autres auteurs et revus par FATIANOFF et GoUET (I969) du moins quand les températures de séchage adoptées sont voisines des nôtres. FatranofF et Gousis (I969) travaillant essentiellement sur des ensilages de luzerne, proposent d'ailleurs pour une température de séchage de $70^{\circ} \mathrm{C}$, des taux de perte respectivement de 54 et $69 \mathrm{p}$. Ioo pour l'ammoniac et les acides gras volatils, alors que nous proposons $53,4 \mathrm{p}$. Ioo et $73,2 \mathrm{p}$. Ioo. Nous avons pu expliquer en partie les variations du taux de pertes des différents composés d'un ensilage à l'autre. Elles sont liées essentiellement au $\mathrm{pH}$ et à la teneur en acides qui déterminent les proportions d'ammoniaque et d'acides gras volatils libres et sous forme de sels plus ou moins sujets à la volatilisation. C'est ainsi que l'ammoniaque est plus volatil lorsque le $\mathrm{pH}$ augmente et que la teneur en acides diminue. De même, les acides gras volatils (notamment l'acide acétique qui est le seul que nous avons pu étudier en détail) sont plus volatils lorsque leur teneur ou celle de l'acide lactique augmente; c'est l'inverse lorsque le $\mathrm{pH}$ de l'ensilage augmente. Cela est dû vraisemblablement au fait que pour des $\mathrm{pH}$ bas, il y a très peu de bases volatiles libres et beaucoup d'acides gras libres alors que c'est l'inverse quand le pH est élevé. Sauf si les ensilages sont très différents de ceux utilisés dans cette étude ( $\mathrm{pH}$ plus faible ou plus élevé) il ne semble cependant pas indispensable de tenir compte de ces variations des coefficients de volatilité en fonction du $\mathrm{pH}$.

L'ammoniaque et les acides gras volatils ne sont cependant pas les seuls composés volatils perdus en quantité importante lors du séchage et c'est pourquoi la méthode de correction proposée par FatianofF et Gouft (I969), basée sur ces 2 seuls composés conduit à une correction insuffisante (tabl. 8); l'acide lactique, qui est en concentration souvent importante dans les bons ensilages, étant partiellement volatil (volatilité de I I à I4 p. Ioo) du moins dans les ensilages de graminées et de maîs. WIL,SON et al. (I964) lui trouvent même un coefficient de volatilité de 34 p. Ioo lorsque le séchage est effectué à $100^{\circ} \mathrm{C}$. Les alcools, volatils à I00 p. Ioo, sont en concentrations très variables et parfois importantes dans les ensilages puisqu'ils peuvent constituer jusqu'à 6 p. Ioo de la matière sèche des ensilages de graminées et 8,5 p. too de celle de certains ensilages de maïs très immatures (cf. tab1. 3). En définitive, la méthode que nous proposons, exigeant moins de travail et beaucoup mieux adaptée aux analyses en série que la méthode au toluène, donne des résultats tout à fait comparables à cette dernière sauf pour les ensilages préparés avec addition d'acide formique. Pour ces derniers, les corrections proposées sous-estiment de Io $\mathrm{g}$ environ par $\mathrm{kg}$ de matière sèche les pertes lors du séchage à l'étuve. Il est vraisemblable que l'acide formique présent dans ces ensilages explique la différence. En effet WAI, 0 ol al. (I97I) trouvent de l'ordre de I3 g d'acide for- 
'IABILEA S

Comparaison des résultats obtenus par différentes méthodes

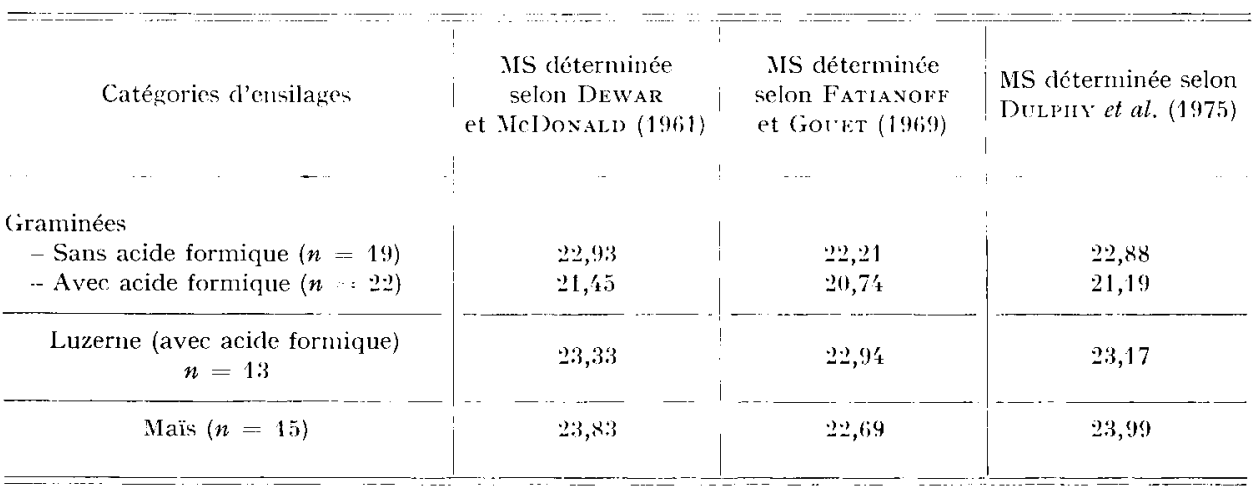

mique par $\mathrm{kg}$ de matière sèche dans 4 ensilages préparés avec addition d'acide formique. Cet acide, plus volatil que l'acide acétique, est pris en compte en même temps que tous les acides lorsqu'on saponifie le distillat dans la méthode au toluène. I1 apparaît donc qu'il faudrait le doser et en tenir compte pour obtenir un bon accord entre la méthode de DEwar et McDonal des ensilages préparés avec de l'acide formique.

Reç pour publication en avril 1975.

\section{SUMMARY}

\section{DETERMINATION OF SILAGE DRY MATTER CONTENT AFTER OVEN-DRYING AND LOSSES OF VOLATILE COMPONENTS}

I. An attempt was made to develop a method for correcting the dry matter content of silages determined after oven-drying, taking into account the various volatile components of these silages and the level of their losses during oven-drying.

2. Comparison was made of the dry matter contents determined after drying in two different ovens, but adjusted at the same temperature $\left(80^{\circ} \mathrm{C}\right)$. The difference between the dry matter contents was very small (on an average o. I I, i.e., $0.52 \mathrm{p}$. Ioo) and negligeable as compared to the accuracy of the determinations of silage cligestibility and voluntary intake in sheep (table 2).

3. Losses of different volatiles were very similar for the two ovens (table 3 ). Therefore, a more detailed study was made on the losses observed during drying in the oven (A) used at the laboratory.

4. Losses of ammonia represented on an average $50.8 \mathrm{p}$. Ioo, but they varied much according to plant species and characteristics of the silages (tables $\&$ and 5). Ammonia was more volatile with increasing $\mathrm{pH}$ levels (grasses and luccrne, table 6).

Drying caused losses of lactic acid in grass and maize silages only. In the latter, the average losses of this acid represented I $2 .+$ p. Ioo (table 4 ). This percentage, although much variable, could not be related to the characteristics of the silages (table 5).

The average losses of acetic acid was $78.7 \mathrm{p}$. Ioo. For grass and lucerne samples, these losses mainly varied with $\mathrm{pH}$ and ammonia content of the silages (tables 5 and 6 ). In the case of maize, they varied with the acetic content itself (table 6). 
The losses of alcohol always represented roo p. Ioo.

For the $6_{5}$ silages studied in details, the amounts lossed of known volatiles represented, on an average, $50.8 \mathrm{~g}$ per $\mathrm{kg}$ non corrected dry matter.

5. On account of these data we suggest, for correction of the dry matter content (determined at $80^{\circ} \mathrm{C}$ ), to add a quantity $\mathrm{Y}$ (volatile components lossed at oven-drying) to $\mathbf{I}$ ooo g "oven dry matter ". The values of $\mathbf{Y}$ are given by the following relations :

$\begin{array}{ll}\text { Grass } & Y=0.616 x_{1}+0.142 x_{2}+0.830 x_{3}+\text { I.000 } x_{4} \\ \text { Lucerne } & Y=0.534 x_{1}+0.732 x_{3}+1.000 x_{4} \\ \text { Maize } & Y=0.382 x_{1}+0.106 x_{2}+0.782 x_{3}+\text { I.000 } x_{4}\end{array}$

in which $\mathrm{Y}$ is the amount of volatile dry matter lossed during oven-drying in $\mathrm{g}$ per $\mathrm{kg}$ non corrected by matter and $x_{1}, x_{2}, x_{3}$ and $x_{4}$ the contents of ammonia, lactic acid, volatile fatty acids and alcohols, respectively, expressed in $\mathrm{g}$ per $\mathrm{kg}$ non corrected dry matter.

6. Our correction method leads to a good concordance between the dry matter contents corrected for losses at oven-drying and the dry matter contents determined by distillation with toluene, except for silages containing formic acid (table 7 and fig. 1 ). For the latter, the value of $Y$ determined by our formulae is about ro $g$ lower than the real losses estimated by comparing the dry matters determined after oven-drying with those obtained after distillation with toluene. This difference is probably largely due to the presence of volatile, but non determined, formic acid. For more exact information, a quantitative determination of this acid would therefore be necessary. The results are discussed.

\section{RÉFÉRENCES BIBL,IOGRAPHIQUES}

Aerts J. V., De Brabander D. L., Cottyn B. G., Buysse F. X., Moermans R. J., i974. Comparison of methods for dry matter determination of high moisture roughages and feces. J. Sci. Fd. Agric. 25, 619-627.

Barker J. B., Summerson W. H., i94I. The coloric determination of lactic acid in biological material. J. Biol. Chem., 137, 535-554.

Brahmakshatriya R. D., Donker J. D., r97I. Five methods for determination of silage dry matter. J. Dairy Sci., 54, I470-I474.

Colovos N. F., Keener H. A., Davis H. A., I957. Errors in drying silage and feces for protein and energy determinations. Improved procedures. J. Dairy Sci., 40, I73-179.

Conway E. J., 1957. Microdiffusion analysis and volumetric error. Crosby, Lokwood, London.

Demarquilly C., I973. Composition chimique, caractéristiques fermentaires, digestibilité et quantité ingérée des ensilages de fourrages. Modifications par rapport au fourrage vert initial. Ann. Zootech., 22, I-35.

Dewar. W. A., McDonald P., i961. Determination of dry matter in silage by distillation with toluene. J. Sci. Fd Agric., 12, 790-795.

Fatianoff N., Gouet Ph., I969. Relation permettant de corriger rapidement et avec précision la matière sèche des ensilages séchés à l'étuve. Ann. Zootech., 18, 407-418.

Fenner H., Barnes H. D., 1965. Improved method for determining dry matter in silage. $J$. Dairy Sci., 48, I324-1 328 .

Larsen R. E., Jones G. M., I973. Effects of different dry matter determination methods on chemical composition and in vitro digestibility of silages. Can. J. Anim. Sci., 53, 753-760.

McDonald P., Dewar W. A., I960. Determination of dry matter and volatiles in silage. J. Sci. Fd Agric., 11, 566-570.

Rigaud J., Journet M., I970. Méthode de dosage des acides gras volatils dans le liquide du rumen. Ann. Biol. anim. Bioch. Biophys., 10, I5 I-157.

Sсносн W., r949. Dje bei der Trecknung von silage proben in Trockenschrank auftretenden verluste an flüchtigen Saüren und Basen. Mitteilungen aus dem Gebeite der Lebensmitteluntersunchung und Hygien, 40, I70-I89.

Waldo D. R., Keys J. E. Jr., Smith L. W., Gordon C. H., I97I. Effect of formic acid on recovery, intake, digestibility and growth from unwilted silage. J. Dairy Sci., 54, 77-84.

Wilson R. F., Tilley J. M. A., Maria Steemers A. Th., I964. Comparison of oven drying and toluene distillation in the determination of the dry matter content of silage. $J$. Sci. Food Agr., 15, I97-200. 